\title{
EFFECTS OF TEAM TIMEOUTS ON THE TEAMS' SCORING PERFORMANCE IN ELITE HANDBALL CLOSE GAMES
}

\author{
Jaime Prieto $^{1}$, Miguel-Ángel Gómez ${ }^{1}$, Anna Volossovitch ${ }^{2}$, and Jaime Sampaio ${ }^{3}$ \\ ${ }^{1}$ Faculty of Physical Activity and Sport Sciences, \\ Polytechnic University of Madrid, Madrid, Spain \\ ${ }^{2}$ SpertLab, CIPER, Faculdade de Motricidade Humana, Universidade de Lisboa, Portugal \\ ${ }^{3}$ Research Center in Sport Sciences, Health and Human Development, \\ University of Trás-os-Montes e Alto Douro at Vila Real, Portugal
}

Original scientific paper

UDC: $796.322: 796.015$

\begin{abstract}
:
This study identified temporal effects (immediate, short-term, and medium-term effect) of team timeouts on elite handball scoring performance in reference to several situational variables that may influence performance: match status, game location, quality of the opposition, game period, and game type. The sample consisted of 646 team timeouts from 142 close games (score differences less than or equal to five goals) of the 2011/2012 men's Spanish Professional Handball League (ASOBAL League). For each team timeout, the goals scored and conceded within the previous and post timeout $1^{\text {st }}, 3^{\text {rd }}$ and $5^{\text {th }}$ ball possessions were recorded for the teams that called a timeout and for their opponents. Multiple linear regression models were used for analysis. The results showed positive effects on goals scored for the teams that called a timeout, with increases of $0.59,1.40$ and 1.85 goals for the periods within the previous and post team timeout $1^{\text {st }}, 3^{\text {rd }}$ and $5^{\text {th }}$ ball possessions. Conversely, significant negative effects on goals scored were found for the opposing teams, with decreases of $0.59,1.43$ and 2.04 goals for the same periods, respectively. Unexpectedly, the influence of situational variables on scoring performance of the teams was only registered for certain game periods. These findings could be useful for a better team timeout management during games and competitions.
\end{abstract}

Key words: match analysis, dynamic perspective, time-dependent analysis, multiple linear regression, coaching science

\section{Introduction}

Handball is a high-scoring sport where teams try to finish all their ball possessions with a shot on goal (and, ideally, convert the shot into a score), because otherwise the referees may penalize passive play and thereby a team may lose possession of the ball (IHF, 2010). A great number of shots leads to the appearance of multiple scoring dynamics along the game (Dumangane, Rosati, \& Volossovitch, 2009). Handball coaches may use different tools to try to change scoring dynamics (usually if it does not favour their team). These tools include verbal instructions given from the bench, player substitutions, and team timeouts (TTO; a 1-minute strategic game interruption that the coach of the attacking team may request). The international handball regulation (IHF, Rules of the game 2010, updated in 2012, rule 2, art. 10) allows national federations to apply different regulations regarding the number of timeouts that teams can request, establishing that each team can benefit from three 1-minute timeouts per game (overtime excluded), but may be granted the maximum of two timeouts in each half of a game. The Royal Spanish Handball Federation applies the latter regulation within the Professional Handball League (ASOBAL League) since the season 2011/2012. Therefore, a change must have been made on the previous regulation, in which the teams were allowed to have only one timeout in each half of a game (i.e. the maximum of two timeouts per team and game). The change underlines the importance of these strategic game breaks as a tool for game management.

Requesting a team timeout constitutes a decision that the coach has to make during the course of the game, thus positioning game analysis within a new promising dynamic time-dependent perspective for modelling sports performance (Glazier, 2010). The dynamic perspective allows the dimension of time to be addressed in order to understand 
continuous dynamic interactions between players and teams (Pfeiffer \& Perl, 2006). In particular, it enables to reveal temporal effects of game actions (i.e. in the short-, medium- or long-term). However, existing literature on handball match analysis from the dynamic approach is very scarce in comparison with the classic static perspective, in which actions are registered with no regard to the game progress over time (for a comprehensive review, see Volossovitch, 2013).

A timeout request can be considered as one of the most important tools in team sports management that allows coaches to provide direct instructions to their players (Bar-Eli \& Tractinsky, 2000; Sampaio, Lago-Peñas, \& Gómez, 2013). Instructions given by coaches during timeouts have been studied from different perspectives, such as critical plays, indications for starters or substitutes, last minutes of the games, and timeout decisions (Gómez, Jiménez, Navarro, Lago-Peñas, \& Sampaio, 2011). Moreover, coaches' cognitive abilities when making decisions during a competition (including timeouts) have been described in several team sports, such as basketball (Bar-Eli \& Tractinsky, 2000), volleyball (Zetou, Kourtesis, Giazitzi, \& Michalopoulou, 2008), and also handball (Debanne, Angel, \& Fontayne, 2014; Debanne \& Fontayne, 2009). Quality of these decisions may be decisive in the course and outcome of the game, thus reflecting coaches' team management skills (Horton, Baker, \& Deakin, 2005).

The main reasons for a timeout calling in handball are: i) to cut down the scoring streak of the opposing team; ii) to adjust, adapt or change the game strategy; iii) to give individual instructions to players; and iv) to enable short recovery from physical and psychological fatigue (Gomes, Volossovitch, \& Ferreira, 2014). Therefore, aspects of multiple areas are involved within a timeout request (e.g. tactics, strategy, physical aspects, and psychological aspects). Interestingly, both opposing teams have an opportunity to take simultaneous advantage of this strategic break (Saavedra, Mukherjee, \& Bagrow, 2012). The coach's decision to call a timeout as well as his/her subsequent instruction is related to the contextual factors (Gomes, et al., 2014). These factors refer to different game conditions that can affect teams and players' performance and are known as situational variables. The analysis of situational variables is a key aspect to be considered when assessing sports performance. This research topic includes match status, game location, quality of opposition, game period, and game type (to get insight into situational variables, see Gómez, Lago-Peñas, \& Pollard, 2013). In this regard, close games (games that end with a small final goal difference) represent the game context where the opposing teams perform at a very similar level, thus equalling the possibility of either team's victory and defining the degree of overall competitiveness of a sport competition (DeSaá, et al., 2012; Meletakos \& Bayios, 2010). However, published research with close handball games in focus is quite scarce (Gómez, Lago-Peñas, Viaño, \& GonzálezGarcía, 2014; Oliveira, Gómez, \& Sampaio, 2012).

Few studies have analysed the timeout effects on teams' performance in sport games. In basketball, research has shown that timeouts appear to be effective in reducing the opponents' rate of reinforcement (Mace, Lalli, Shea, \& Nevin, 1992; Roane, Kelley, Trosclair, \& Hauer, 2004). Gómez et al. (2011) studied 144 timeouts from the European Basketball Championship (Spain, 2007). The results showed that offensive and defensive performances were better after a timeout than before, with better offensive performances in the last five minutes of the games. Sampaio et al. (2013) analysed a sample of 60 games of the men's Spanish Professional Basketball League. The authors found positive effects on the points scored for the teams that called the timeout and negative effects for the opponents. In handball, Gomes et al. (2014) focused on identifying the different contexts of a team timeout calling in a sample of 2,178 timeouts from the Spanish ASOBAL League. The authors described different contexts in which the team timeouts (hereinafter referred to as TTO) were called by the teams, including match status, game location, and game period, as well as short-term offensive and defensive teams' performance. However, this only existing study in handball did not analyse possible effects of TTOs on the subsequent teams' performance. Besides, the statistical model did not account for a multivariate model that allow for interactive effects description and prediction based on the goals scored/conceded. As pointed out in the existing literature, accounting for the interactive nature of potential competition constraints appears critical to competition-specific preparation due to the complex and dynamic nature of high-level team sports performance, including handball (Eccles, Ward, \& Woodman, 2009; McGarry \& Franks, 2003). In this sense, more powerful statistical procedures should strengthen the analysis of the relationships between the game-environment variables, which may involve high-order complex interactions (Garganta, 2009). In particular, the use of multivariate techniques might be useful for the description and prediction of the normative profiles of timeouts and their association with situational variables and teams' performance indicators (Moura, Martins, \& Cunha, 2014).

Within this context, the purpose of this study was to analyse possible temporal effects (immediate effect, short-term effect, and medium-term effect) of TTOs on the scoring performance of the teams that called TTO and of their opponents in elite hand- 
ball (ASOBAL League) close games, when controlling for match status, game location, quality of opposition, and game period. We hypothesised that calling TTO would have positive effects on the scoring performance of the teams that called it and would be coupled with negative effects for the opponents' scoring. Moreover, we expected to find significant effects on scoring performances according to match status, game location, quality of opposition, and game period.

\section{Methods}

\section{Sample}

The study focused on the 240 games played in the 2011/2012 season of the men's Spanish Professional Handball League (ASOBAL League). In order to account for the game type, specific margins of victory were considered, conducting a $k$-means cluster analysis that classified the games as follows: unbalanced games (the final score difference in a range from 13 to 25 goals, averaging $15.61 \pm 3.05$ goals, $n=18)$, balanced games ( $6-12$ goals, $8.57 \pm 2.02$ goals, $\mathrm{n}=80$ ), and close games ( $0-5$ goals, $2.23 \pm 1.59$ goals, $n=142$ ). With the aim of controlling for the highest game competitiveness, only close games $(n=142)$ were considered for further analysis (Gómez, et al., 2014; Meletakos \& Bayios, 2010). All the 646 TTOs granted in these close games were registered (an average of 4.55 TTOs per game).

\section{Procedures}

The official play-by-play sheets of the games under analysis were collected from the website of the ASOBAL League (www.asobal.es). Although all the play-by-play data were gathered by professional technicians of the League, a subsample of 64 TTOs ( $10 \%$ of the total) were randomly selected in order to test the reliability of the recorded actions in each TTO situation. The subsequent kappa test showed coefficients of agreement of 1.0 for the goals scored and conceded by the teams.

Data logging for each TTO was divided into two areas. In order to analyse temporal effects of TTOs on the scoring performance of the teams, the number of goals scored before and after each TTO was registered for both opposing teams. Each ball possession was set as the unit for temporal effects analysis (dynamic time-dependent perspective). In particular, the goals scored for the periods within the previous and post TTO first, third and fifth ball possessions were registered. The TTO immediate effects were calculated by the difference between the goals scored and conceded one ball possession before the TTO calling and one ball possession after it. Short-term effects considered the goal differences registered at the third ball possession prior to TTO and the third ball possession after it. Medium- term effects considered the goal differences registered five ball possessions before the TTO and five ball possessions after it. In certain cases some of these data could not be registered, since some (or all) of the considered ball possessions were not played. For example, when two TTO are granted within a small margin of time (e.g. two minutes), often there is not enough time for five ball possessions to be played between them. This also generally happens when a TTO is granted in the last minutes of each half of the game. As a result, the ball possessions registered were as follows - for the teams calling the TTO: $n=634, n=532$ and $n=486$ ball possessions, for the previous and post TTO $1^{\text {st }}$, $3^{\text {rd }}$ and $5^{\text {th }}$ ball possessions, respectively, and for the opposing teams: $n=624, n=524$ and $n=417$ ball possessions, respectively.

Regarding the influence of situational variables on teams' performance, match status, game location, quality of opposition, and game period were registered. The match status was computed as the goal difference between the teams when the timeout was granted (the team is either winning, losing or drawing). The game location distinguished whether the team calling a TTO was playing at home or away (the opposite for the opposing team). The quality of opposition was computed by the difference between the final ranking positions of each pair of the opposing teams. Finally, regarding the game period, the minute in which each TTO was granted was registered. In this sense, according to previous studies (Gomes, et al., 2014), the handball game was divided into six 10 -minute periods: $(0-10 \mathrm{~min}$, i.e. actions from 0:00 to 9:59), (10-20min, i.e. actions from 10:00 to 19:59), (20-30min), (30-40 min), (40$50 \mathrm{~min})$, and (50-60min).

\section{Statistical analysis}

Descriptive data were computed to describe the game situations in which TTOs were called. Multiple linear regression models were applied to identify the effects of the independent situational variables on the scoring performance of the team calling a TTO and of the opponents. The model was adapted from Sampaio et al. (2013) who applied it in basketball. The response variable (dependent variable) of the model was the teams' goal difference (i.e. the difference between goals scored and conceded by each team). Five explanatory variables or regressors (independent variables) were included in the model: TTO, match status, game location, quality of opposition, and game period. The model met the assumptions of distribution normality and linearity between the dependent and independent variables. Furthermore, no heteroscedasticity in residuals or multicollinearity between the regressors was observed when estimating the model. Additionally, the Ramsey (1969) regression equation specifica- 
tion error test (RESET) did not show any specification problems. Thus, the resulting multiple linear regression model with the five regressors, given $N$ observations, is:

$$
\begin{aligned}
& \mathrm{GD}=\beta_{0}+\beta_{1} \bullet \mathrm{TTO}+\beta_{2} \bullet \mathrm{MS}+\beta_{3} \bullet \mathrm{GL}+\beta_{4} \bullet \mathrm{QO} \\
& +\beta_{5} \bullet \mathrm{GP}+\varepsilon
\end{aligned}
$$

where: GD $=$ the difference between team's goals scored and conceded; $\beta_{0}=$ intercept of the regression (often referred to as the constant); $\beta_{i}=$ the effects or regression coefficients of the regressors; TTO = timeout, a dummy variable stating whether the goals were scored and conceded before $(\mathrm{TTO}=$ $0)$ or after $(\mathrm{TTO}=1)$ the TTO; MS = match status, $\mathrm{GL}$ = game location, $\mathrm{QO}=$ quality of opposition, $\mathrm{GP}$ $=$ game period, $\varepsilon=$ disturbance term (also referred as error term or noise). These multiple linear regression analyses were performed for the periods within the first, third and fifth ball possessions before and after TTO requests. When interpreting the results of the model, positive or negative coefficients indicate a greater or lower propensity to increase or decrease the teams' goal differences. Statistical significance was set at $\mathrm{p} \leq .05$. Statistical analyses were performed using SPSS 21.0 (IBM Corp., Armonk, NY, USA).

\section{Results}

Descriptive data regarding the distribution of TTOs within different game contexts are presented in Figure 1. The results show an unequal distribution of TTOs in different game periods according to the match status. The majority of TTOs were requested during the last 10 minutes of each half of the game ( $60.06 \%$ of the total of TTOs; $26.01 \%$ in the period $20-30 \mathrm{~min} ; 34.05 \%$ in $50-60 \mathrm{~min}$ ). In addition, an increasing trend in the TTO request is revealed as the game progresses throughout each half of the game $3.87 \%$ of the total TTOs in the period $0-10 \mathrm{~min}, 13.00 \%$ in $10-20 \mathrm{~min}, 26.01 \%$ in $20-30 \mathrm{~min} ; 7.74 \%$ in $30-40 \mathrm{~min}, 15.33 \%$ in $40-50 \mathrm{~min} ; 34.06 \%$ in $50-60 \mathrm{~min})$. Regarding the match status, $55.88 \%$ of the total TTOs were requested by the teams when they were losing, $32.66 \%$ when they were winning and $11.46 \%$ were called when the game was in a tie. In particular, it should be noted that the majority of TTOs in the first 20 minutes of the game were called by the teams when they were losing $(100.00 \%$ in the period $0-10 \mathrm{~min} ; 88.10 \%$ in the period $10-20 \mathrm{~min})$; the trend decreased as the game progressed in each of its halves. So, in the last 10 minutes of each half, the number of TTOs was shown quite even between the losing and the winning context for the team calling a TTO $(44.64 \%$ of the TTOs requested when losing and $48.21 \%$ when winning in the period $20-30 \mathrm{~min}$ and respective $44.55 \%$ and
$36.36 \%$ for the period $50-60 \mathrm{~min})$. With respect to the game location, the results showed that home and away teams called mostly the same number of TTOs (49.85\% of the total TTOs requested by local teams; $50.15 \%$ requested by away teams). Figure 2 shows the means and standard deviations in goals scored by the teams calling a TTO and by the opposing teams in the previous and post analysed ball possessions.

The multiple linear regression analysis (Table 1) showed significant positive effects of TTOs called on the number of goals scored for the teams calling a TTO, with increases of $0.59,1.40$ and 1.85 goals for the periods within the previous and post TTO $1^{\text {st }}, 3^{\text {rd }}$ and $5^{\text {th }}$ ball possessions (all $\mathrm{p} \leq .05$ ). Conversely, significant negative effects were found for

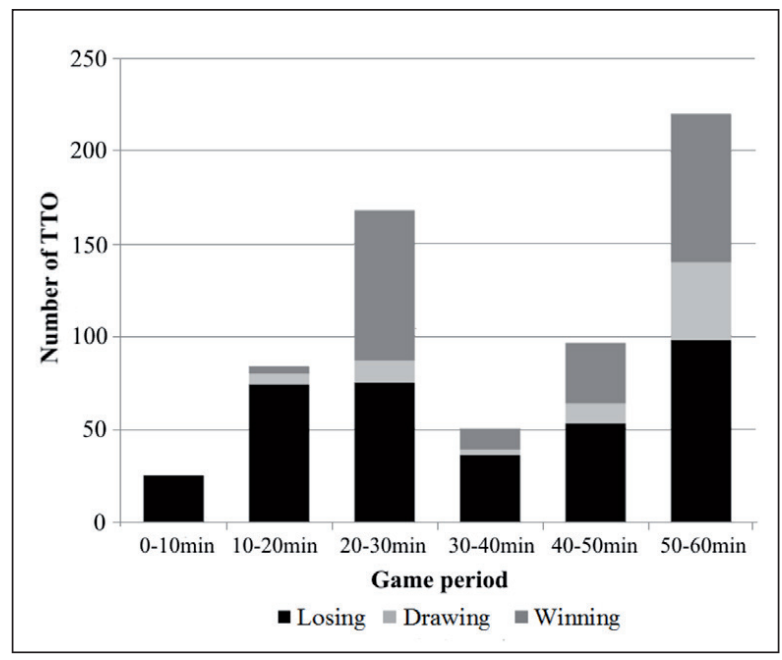

Figure 1. Distribution of TTOs in the different game periods according to the match status. The division of the bars shows whether the team that called a TTO was losing, drawing or winning the game.

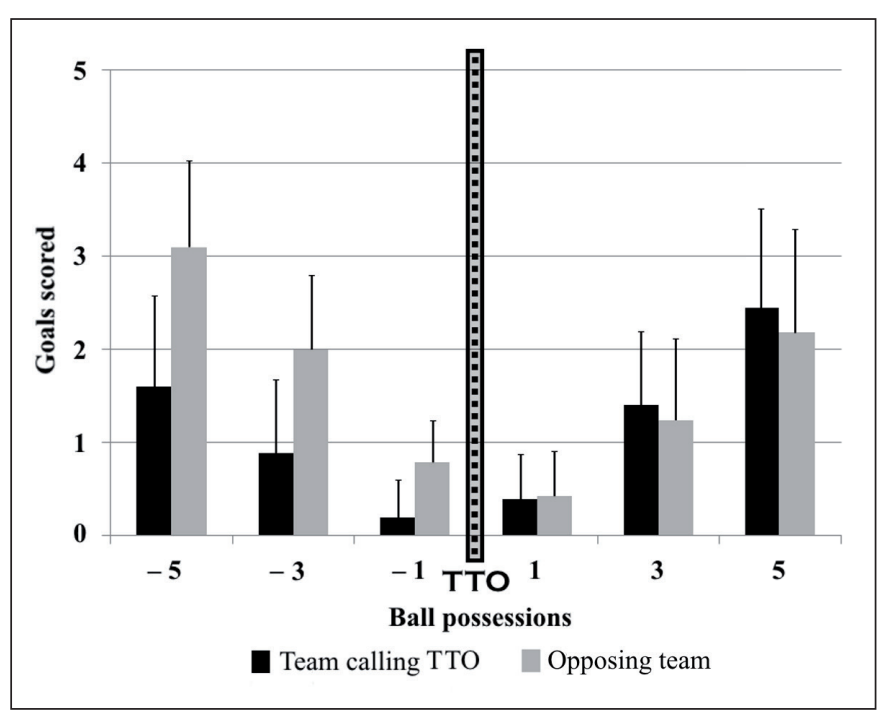

Figure 2. Goals scored by the teams that called a TTO and by the opposing teams in the previous and post TTO analysed ball possessions. The thick dashed vertical line represents a TTO. The thin vertical lines represent the standard deviations. 
Table 1. Effects of the independent variables on the team's goal differences according to the number of ball possessions for the teams that called a TTO and for the opposing teams. Standard errors are presented in parentheses

\begin{tabular}{|c|c|c|c|c|c|c|c|c|c|c|c|c|}
\hline \multirow{4}{*}{$\begin{array}{l}\text { Variables } \\
\text { Timeout }\end{array}$} & \multicolumn{12}{|c|}{ Ball possessions } \\
\hline & \multicolumn{4}{|c|}{$-1 / 1$} & \multicolumn{4}{|c|}{$-3 / 3$} & \multicolumn{4}{|c|}{$-5 / 5$} \\
\hline & \multicolumn{2}{|c|}{$\begin{array}{c}\text { Team calling } \\
\text { TTO }\end{array}$} & \multicolumn{2}{|c|}{ Opposing team } & \multicolumn{2}{|c|}{$\begin{array}{c}\text { Team calling } \\
\text { TTO }\end{array}$} & \multicolumn{2}{|c|}{ Opposing team } & \multicolumn{2}{|c|}{$\begin{array}{c}\text { Team calling } \\
\text { TTO }\end{array}$} & \multicolumn{2}{|c|}{ Opposing team } \\
\hline & $0.59 *$ & $(0.04)$ & $-0.59^{*}$ & $(0.04)$ & $1.40^{*}$ & $(0.07)$ & $-1.43^{*}$ & $(0.07)$ & $1.85^{*}$ & $(0.09)$ & $-2.04^{*}$ & $(0.10)$ \\
\hline Match status & -0.00 & $(0.01)$ & -0.00 & $(0.01)$ & 0.00 & $(0.01)$ & -0.00 & $(0.01)$ & 0.03 & $(0.02)$ & -0.02 & $(0.02)$ \\
\hline $\begin{array}{l}\text { Game } \\
\text { location }\end{array}$ & -0.03 & $(0.04)$ & -0.03 & $(0.04)$ & -0.09 & $(0.07)$ & -0.09 & $(0.07)$ & -0.03 & $(0.10)$ & -0.07 & $(0.10)$ \\
\hline $\begin{array}{l}\text { Quality of } \\
\text { opposition }\end{array}$ & -0.01 & $(0.00)$ & -0.01 & $(0.00)$ & -0.00 & $(0.01)$ & 0.00 & $(0.01)$ & -0.00 & $(0.01)$ & -0.01 & $(0.01)$ \\
\hline \multicolumn{13}{|l|}{ Game period } \\
\hline \multicolumn{13}{|l|}{ First half } \\
\hline 0-10min & 0.16 & $(0.10)$ & -0.16 & $(0.10)$ & 0.08 & $(0.18)$ & -0.07 & $(0.18)$ & $0.48^{*}$ & $(0.23)$ & -0.06 & $(0.23)$ \\
\hline $10-20 \min$ & $0.13^{*}$ & $(0.07)$ & $-0.13^{*}$ & $(0.07)$ & $0.27^{*}$ & $(0.12)$ & $-0.26^{*}$ & $(0.12)$ & $0.45^{\star}$ & $(0.16)$ & -0.01 & $(0.16)$ \\
\hline $20-30 \mathrm{~min}$ & a & & a & & a & & a & & a & & 0.22 & $(0.15)$ \\
\hline \multicolumn{13}{|l|}{ Second half } \\
\hline $30-40 \mathrm{~min}$ & $0.25^{*}$ & $(0.08)$ & $-0.25^{*}$ & $(0.08)$ & $0.53^{*}$ & $(0.14)$ & $-0.51^{*}$ & $(0.14)$ & $0.70^{*}$ & $(0.18)$ & -0.24 & $(0.18)$ \\
\hline $40-50 \min$ & $0.12^{*}$ & $(0.06)$ & -0.12 & $(0.06)$ & 0.18 & $(0.11)$ & -0.16 & $(0.11)$ & $0.51^{*}$ & $(0.14)$ & a & \\
\hline $50-60 \mathrm{~min}$ & -0.04 & $(0.05)$ & 0.05 & $(0.05)$ & -0.04 & $(0.10)$ & 0.04 & $(0.01)$ & 0.14 & $(0.13)$ & 0.19 & $(0.15)$ \\
\hline Intercept & $-1.18^{*}$ & $(0.21)$ & $1.19^{*}$ & $(0.22)$ & $-2.09^{*}$ & $(0.40)$ & $2.16^{*}$ & $(0.40)$ & $-3.45^{*}$ & $(0.52)$ & $1.79^{*}$ & $(0.54)$ \\
\hline $\begin{array}{l}\text { Number of } \\
\text { observations }\end{array}$ & \multicolumn{2}{|c|}{634} & \multicolumn{2}{|c|}{624} & \multicolumn{2}{|c|}{532} & \multicolumn{2}{|c|}{524} & \multicolumn{2}{|c|}{486} & \multicolumn{2}{|c|}{417} \\
\hline $\mathrm{R}^{2}$ & \multicolumn{2}{|c|}{.18} & \multicolumn{2}{|c|}{.18} & \multicolumn{2}{|c|}{.29} & \multicolumn{2}{|c|}{.30} & \multicolumn{2}{|c|}{.29} & \multicolumn{2}{|c|}{.35} \\
\hline
\end{tabular}

$* p \leq .05$

Note $=$ a: excluded values from linear regression equation

the opposing teams, with decreases of $0.59,1.43$ and 2.04 goals for the periods within the previous and post TTO $1^{\text {st }}, 3^{\text {rd }}$ and $5^{\text {th }}$ ball possessions (all $\mathrm{p} \leq .05$ ). In order to translate these effects into goals per possession, the results were divided by the number of the elapsed ball possessions. For the teams that called a TTO the increases were of $0.59,0.47$ and 0.37 goals per possession for the first, third and fifth ball possessions, respectively; while for the opposing teams the decreases were of $0.59,0.48$ and 0.41 goals per ball possession, respectively.

Match status, game location, and quality of opposition did not show any significant effects on the change in goal difference before and after a TTO, either for the calling teams or for the opponents. At the same time, the model parameters indicated that the effect of TTOs on the goals scored during the ball possessions under analysis varied according to the game period (see Table 1). In particular, positive effects were found for the teams calling a TTO within the second, fourth and fifth game periods in reference to both the previous and post TTO first ball possession (i.e. immediate effects), associated to increases in goals scored by the teams $(0.13,0.25$, and 0.12 goals, respectively), whereas negative effects were found in the second and fourth game period, with a decrease of
0.13 and 0.25 goals, respectively. Similarly, positive effects were found for the teams calling a TTO in the second and fourth game period with regard to previous and post TTO third ball possessions (i.e. short-term effects), associated to an increase in the goals scored $(0.27$ and 0.53 goals, respectively), coupled with negative effects for the opposing teams in the same situations $(-0.26$ and -0.51 goals). Finally, positive effects were found for the teams that called a TTO in the periods comprising the first 20 minutes of each half of the game (i.e. 0-10min, 10-20min, 30-40min, 40-50 min) when analysing the previous and post TTO fifth ball possessions (i.e. medium-term effects), associated to an increase in the goals scored by the teams $(0.48,0.45$, 0.70 , and 0.51 goals, respectively). No significant negative effects on the goals scored in the previous and post TTO fifth ball possessions were observed for the opposing teams. The intercept of the regressions was significant in all the models (all $\mathrm{p} \leq .05$ ).

\section{Discussion and conclusions}

In this study, multiple linear regression models were used to investigate temporal effects of TTOs on the teams' scoring performance in elite handball close games, which represent the highest level of game competitiveness. 
With respect to the distribution of TTOs, the results are consistent with the recent study by Gomes et al. (2014) on three seasons of the ASOBAL League, in which $57 \%$ of TTOs were called in the last 10 minutes of each half $(34 \%$ and $23 \%$ respectively). The results also support evidence from the 2004 and 2008 European Handball Championships (Prudente, Lopes, \& Fernando, 2009; Sevim \& Traborsky, 2004). All these studies analysed all types of games, not only the close ones. Hence, it may be suggested that the majority of TTOs in handball are called in the last 10 minutes of each half of the game, regardless of the game type. The increase in the number of TTOs called as the game progresses in each of its halves may be related to the coaches' strategy to delay the TTO request in order to use this strategic break at the critical moments that frequently occur in the last minutes of the game, as was argued in previous research in basketball (Gómez, et al., 2011; Kozar, Whitfield, Lord, \& Mechikoff, 1993). With respect to the match status, the results suggest that the first TTO in handball close games is requested to stop an opponent's scoring streak, or, at least, to interrupt the own team's continued negative performance. As the game progresses, TTOs are called by the teams also when they are winning or the game is in a tie. These results are consistent with Gomes et al. (2014). In line with Kozar et al. (1993) in basketball, Gomes et al. (2014) argued that the natural development of the game itself makes coaches not to let go the opportunity to use TTOs they can call in each half of the game, either for trying to invert the unfavourable current result or to enhance score advantage. With respect to the game location, the results suggest that in handball close games the home and away teams request almost the same number of TTOs. These results are partly consistent with those obtained by Gomes et al. (2014) when analysing balanced score situations.

As was hypothesised, the results show that the teams calling a TTO increased their scoring performance immediately, in the first possession at their disposal after TTO (immediate effect), but also in short- and medium-term (periods within the previous and post TTO third and fifth ball possessions, respectively), whereas the opposing teams decreased their scoring within the same ball possessions they had after a TTO. These findings are partly consistent with previous results in basketball reported by Sampaio et al. (2013), in which positive effects on the scoring were found for the teams that called a TTO, whereas the opponents decreased their points scored in the same situations, ceasing the effect at some point from the fifth to the tenth ball possession (i.e. the authors studied the previous and post timeout 3,5 and 10 ball possessions). This point is difficult to compare due to different game development features of the game of handball and basketball, mainly associated with the duration of ball possessions (i.e. not strictly limited time in handball vs. exact 24 seconds or less in basketball). Very interestingly, when dividing the data by the number of elapsed ball possessions (goals per possession), we can see that the obtained positive and negative effects decreases as ball possessions go by. This trend suggests that the effect of TTO on teams' scoring performance per ball possession in handball, both positive and negative, is greater as time is closer to the halftime, but decreases as more ball possessions elapse. That is, the TTO effect is maximum in the immediate term, but it decreases progressively in the shortand medium-term, although remaining still significant in the post TTO fifth ball possession. As previously stated in literature (Duke \& Corlett, 1992; Smisson, Burke, Joyner, Munkasy, \& Blom, 2007), multiple causes might explain the timeout effects in team sports, although they were very difficult to isolate and quantify (Sampaio, et al., 2013). These include strategic, technical, physical and psychological aspects that either allow, or inhibit, players and teams to regain control over the game through the maintenance or recovery of the tactical order and/or motor and mental control (Duke \& Corlett, 1992; Kozar, et al., 1993). In all these situations, the role of coaches and their new, or repeated, instructions they give to players during a TTO are fundamental. In particular, the possible psychological advantage gained by interrupting the opponents' positive performance (e.g. due to a timeout break) has been suggested as a possible extra psychological empowerment, which might alter interpersonal perceptions and influence players' physical and mental performances (Iso-Ahola \& Mobily, 1980; Roane, et al., 2004). However, theorists still appear to be divided on whether this concept is real or fictitious, due to the lack of consistent evidence regarding the relationship between perceived momentum and actual performance (for a comprehensive review, see Crust \& Nesti, 2006). In fact, experimental studies showed evidence that perceptions of negative momentum lead to performance improvement (e.g. Stanimirovic \& Hanrahan, 2004). In a recent study, Moescha, Bäckström, Granér, and Apitzsch (2014) found no significant signs for momentum in female elite handball games, with some of them even showing signs for anti-momentum, but uncovered that five-minute periods of momentum happen in nearly $75 \%$ of all games, leading to the hypothesis that handball players base their belief in momentum on short-term periods. This finding is very interesting and opens a door to the investigation if the experience of short time breaks in the game, such as TTOs, is perceived by the players as an indicator of momentum during the game.

Surprisingly and contrary to the expected, our results showed no significant effects of TTOs on teams' scoring performance according to the match 
status, game location, and quality of opposition, neither for the teams that called a TTO nor for their opponents. These results are consistent with those obtained by Sampaio et al. (2013) in basketball. Hence, it may be suggested that in handball close games, scoring performance of two opposing teams after a TTO is similarly affected by match status, game location, and quality of opposition. This result is very intriguing and different from much research highlighting the influence of situational variables in team sports performance (e.g. Gómez, et al., 2011; Sampaio, Lago, Casais, \& Leite, 2010). In this connection, it has been suggested that the adjusted competitive level existing in close games could mask the effect of the mentioned situational variables, although research on this point is still inconclusive (Gómez, Lorenzo, Ibáñez, \& Sampaio, 2013; Oliveira, et al., 2012).

With respect to the game period, medium-term significant positive effects (i.e. within the previous and post TTO fifth ball possessions) were found for the teams that called a TTO in the periods comprising the first 20 minutes of each half of the game, associated to an increase in the goals scored. In particular, the increase in the goals scored for the teams calling a TTO in the initial 20 minutes of the game, coupled with the fact that the majority of TTOs in these periods were requested by the teams when they were losing, suggests that if these TTOs had not been called, these games might have more likely been unbalanced. Therefore, this may mean that early game periods are best for TTO calling when losing in handball close games. However, a specific design of the study plays an important role here (i.e. game period distribution, close games cataloguing, etc.), thus opening doors to future research in this direction but with different parameters. Nevertheless, internal logic of the game suggests that some of TTOs should be saved for the final phases of each half of the games. In this line, Bar-Eli, Tenenbaum, and Elbaz (1990) stated that the final phase of handball game was the most critical one. Conversely, in basketball, periods at the beginning of the games have been also identified as critical moments (Sampaio, et al., 2010), thus opening new avenues for future research in handball.

In summary, the current results suggest that in elite handball close games calling a TTO has positive effects on the scoring performance of the calling teams, coupled with negative effects on the goals scored for their opponents. The influence of situational variables is only reflected in relation to certain game periods. From a practical perspective, handball coaches could apply these findings for a better TTO management. In this sense, training suggestions could include: i) designing practice tasks simulating different competitive environments for a timeout request, trying to define specific tactics and/or plays to perform after the game stoppage in all the possible game contexts; ii) training the timeout contents with the aim of establishing the most direct and effective communication among coaches and players; iii) specifically during timeouts, coaches should remind their players about specific trained moves and/or tactics for each game context, focus on strategy for upcoming play and/or give individual instructions to their players; and iv) within the subsequent analysis of the games, coaches and team technicians should examine each TTO in order to assess their effectiveness and try to improve it for upcoming games. Future research could investigate TTO effects from this time-dependent dynamic perspective in other handball competitive contexts (other types of games, other leagues, international championships, women's handball) and contrast them with the present results. In addition, it would be worthy to analyse if a better post TTO scoring performance has an effect on the final outcome of the game (not only in terms of previous and post TTO ball possessions), that is, whether the teams that manage better post TTO ball possessions win more games. Furthermore, adapting this type of study to other sports where TTOs are granted also has potential for the future.

\section{References}

Bar-Eli, M., Tenenbaum, G., \& Elbaz, G. (1990). Psychological strain in competition: The role of time phases. Sportwissenschaft, 20, 182-191.

Bar-Eli, M., \& Tractinsky, N. (2000). Criticality of game situations and decision making in basketball: An application of performance crisis perspective. Psychology of Sport and Exercise, 1, 27-39.

Crust, L., \& Nesti, M. (2006). A review of psychological momentum in sports: Why qualitative research is needed. Athletic Insight Journal, 8, 1-15.

Debanne, T., Angel, V., \& Fontayne, P. (2014). Decision-making during games by professional handball coaches using regulatory focus theory. Journal of Applied Sport Psychology, 26(1), 111-124.

Debanne, T., \& Fontayne, P. (2009). A study of successful experienced elite handball coach's cognitive processes in competition situations. International Journal of Sports Science \& Coaching, 4, 1-16. 
DeSaá, Y., Martín, J.M., Sarmiento, S., Rodríguez, D., García-Rodríguez, A., \& García-Manso, J.M. (2012). A model for competitiveness level analysis in sports competitions: Application to basketball. Physica A: Statistical Mechanics and its Applications, 391(10), 2997-3004.

Duke, A., \& Corlett, J. (1992). Factors affecting university women's basketball coaches' timeouts decisions. Canadian Journal of Sports Sciences, 17, 333-337.

Dumangane, M., Rosati, N., \& Volossovitch, A. (2009). Departure from independence and stationarity in a handball match. Journal of Applied Statistics, 36(7), 723-741.

Eccles, D.W., Ward, P., \& Woodman, T. (2009). The role of competition-specific preparation in expert sport performance. Psychology of Sport and Exercise, 10, 96-107.

Garganta, J. (2009). Trends of tactical performance analysis in team sports: Bridging the gap between research, training and competition. Revista Portuguesa de Ciencias Desporto, 9(1), 81-89.

Glazier, P.S. (2010). Game, set and match? Substantive issues and future directions in performance analysis. Sports Medicine, 40(8), 625-634.

Gomes, F., Volossovitch, A., \& Ferreira, A.P. (2014). Team timeout calling in handball. International Journal of Performance Analysis in Sport, 14(1), 98-110.

Gómez, M.A., Jiménez, S., Navarro, R., Lago-Peña, C., \& Sampaio, J. (2011). Effects of coaches’ timeouts on basketball teams' offensive and defensive performances according to momentary differences in score and game period. European Journal of Sport Science, 11(5), 303-308.

Gómez, M.A., Lago-Peñas, C., \& Pollard, R. (2013). Situational variables. In T. McGarry, P. O’Donoghue \& J. Sampaio (Eds.), Routledge handbook of sport performance analysis (pp. 259-269). London: Routledge.

Gómez, M.A., Lago-Peñas, C., Viaño, J., \& González-García, I. (2014). Effects of game location, team quality and final outcome on game-related statistics in professional handball close games. International Journal of Performance Analysis in Sport, 46(2), 249-257.

Gómez, M.A., Lorenzo, A., Ibáñez, S., \& Sampaio, J. (2013). Ball possession effectiveness in men's and women's elite basketball according to situational variables in different game periods. Journal of Sports Sciences, 14, 1578-1587.

Horton, S., Baker, J., \& Deakin, J. (2005). Experts in action: A systematic observation of 5 national team coaches. International Journal of Sport Psychology, 36, 299-319.

International Handball Federation. (2010). Rules of the game. International Handball Federation. Retrieved $17^{\text {th }}$ October, 2014 from http://www.ihf.info/upload/PDF-Download/rules_english.pdf

Iso-Ahola, S., \& Mobily, K. (1980). Psychological momentum: A phenomenon and an empirical (unobtrusive) validation of its influence in a competitive sport tournament. Psychological Reports, 46, 391-401.

Kozar, B., Whitfield, K.E., Lord, R.H., \& Mechikoff, R.A. (1993). Timeouts before free-throws: Do the statistics support the strategy? Perceptual and Motor Skills, 76, 47-50.

Mace, F., Lalli, J., Shea, M., \& Nevin, J. (1992). Behavioral momentum in college basketball. Journal of Applied Behavior Analysis, 25, 657-663.

McGarry, T., \& Franks, I. (2003). The science of match analysis. In T. Reilly \& M. Williams (Eds.), Science and soccer (pp. 265-275). London: Routledge.

Meletakos, P., \& Bayios, I. (2010). General trends in European men's handball: A longitudinal study. International Journal of Performance Analysis in Sport, 10(3), 221-228.

Moescha, K., Bäckströma, K., Granéra, S., \& Apitzscha, E. (2014). Hard fact or illusion? An investigation on momentum in female elite handball from a team perspective. International Journal of Sport and Exercise Psychology, 12(2), 106-120.

Moura, F.A., Martins, L.E.B., \& Cunha, S.A. (2014). Analysis of football game-related statistics using multivariate techniques. Journal of Sports Sciences, 32(20), 1881-1887.

Oliveira, T., Gómez, M.A., \& Sampaio, J. (2012). Effects of game location, period and quality of opposition in elite handball performances. Perceptual and Motor Skills, 114(3), 783-794.

Pfeiffer, M., \& Perl, J. (2006). Analysis of tactical structures in team handball by means of artificial neural networks. International Journal of Computer Science in Sport, 5(1), 4-14.

Prudente, J., Lopes, H., \& Fernando, C. (2009). O time-out no andebol: análise da tomada de decisão do treinador na utilização do desconto de tempo durante a competição no Campeonato da Europa de 2008. [Team timeout in handball: Coach's decision-making analysis during the 2008 European Championship. In Portuguese.] In II Congreso Internacional de Deportes de Equipo, Coruña. Retrieved 17 $7^{\text {th }}$ October 2014 from http://www. altorendimiento.com/es/congresos/balonmano/46-o-time-out-noandebol-analise-da-tomada-de-decisao-dotreinador-na-utilizacao-do-descontode- tempo-durante-a-competicao-no-campeonato-da-europa-de-2008

Ramsey, J. (1969). Test for specification errors in classical lineal least squares regression analysis. Journal of the Royal Statistical Society, 31, 350-371.

Roane, H., Kelley, M., Trosclair, N., \& Hauer, L. (2004). Behavioral momentum in sports: A partial replication with women's basketball. Journal of Applied Behavior Analysis, 37, 385-390.

Saavedra, S., Mukherjee, S., \& Bagrow, J.P. (2012). Is coaching experience associated with effective use of timeouts in basketball? Nature - Scientific Reports, 2, doi: 10.1038/srep00676. 
Sampaio, J., Lago, C., Casais, L., \& Leite, N. (2010). Effects of starting score-line, game location, and quality of opposition in basketball quarter score. European Journal of Sports Sciences, 10(6), 391-396.

Sampaio, J., Lago-Peñas, C., \& Gómez, M.A. (2013). Brief exploration of short- and mid-term timeout effects on basketball scoring according to situational variables. European Journal of Sport Science, 13(1), 25-30.

Sevim, Y., \& Taborsky, F. (2004). Qualitative trend analysis of the 6th Men's European Championship Slovenia 2004. Euro Handball. Retrieved $17^{\text {th }}$ October 2014 from: http://home.eurohandball.com/ehf_files/specificHBI/ECh Analyses/2004/slo/4/Sevim_EURO2004_Trendanalysis.pdf

Smisson, C., Burke, K., Joyner, B., Munkasy, B., \& Blom, L. (2007). Spectators' perceptions of momentum and personal control: Testing the antecedents-consequences model. Athletic Insight, 9, 79-90.

Stanimirovic, R., \& Hanrahan, S.J. (2004). Efficacy, affect, and teams: Is momentum a misnormer? International Journal of Sport and Exercise Psychology, 2, 43-62.

Volossovitch, A. (2013). Handball. In T. McGarry, P. O’Donoghue \& J. Sampaio (Eds.), Routledge handbook of sport performance analysis (pp. 380-392). London: Routledge.

Zetou, E., Kourtesis, T., Giazitzi, K., \& Michalopoulou, M. (2008). Management and content analysis of timeouts during volleyball games. International Journal of Performance Analysis in Sport, 8, 44-55.

Submitted: May 14, 2015

Accepted: October 19, 2015

Correspondence to:

Jaime Prieto

Faculty of Physical Activity and Sport Sciences

Polytechnic University of Madrid

Martín Fierro, 7, 28040 Madrid, Spain

Phone: +34667994837

E-mail: jaimeprietobermejo@gmail.com 